\title{
THE WAVE, LAPLACE, AND HEAT EQUATIONS AND RELATED TRANSFORMS
}

\author{
by J. W. DETTMAN $\dagger$
}

(Received 12 April, 1969)

1. Introduction. This paper is concerned with three basic transforms:

$$
\begin{aligned}
& f(t)=\frac{1}{\sqrt{(\pi t)}} \int_{0}^{\infty} e^{-s^{2} / 4 t} g(s) d s, \\
& f(t)=\frac{2 t}{\pi} \int_{0}^{\infty} \frac{g(s)}{s^{2}+t^{2}} d s, \\
& f(t)=\frac{t}{\sqrt{ }(4 \pi)} \int_{0}^{\infty} e^{-t^{2} / 4 s} s^{-3 / 2} g(s) d s .
\end{aligned}
$$

The first of these has been studied by Widder [1], who points out that $f(t)$ can be interpreted as the temperature $u(0, t)$ on the time axis, where $u(x, t)$ is the solution of the heat equation with symmetric initial temperature $u(x, 0)=g(|x|)$. The second has also been studied by Widder [2], where it is pointed out that $f(t)$ can be interpreted as the value of the harmonic function $u(x, t)$ on the $t$-axis arising from the boundary data $u(x, 0)=g(|x|)$. In view of some recent work by L. R. Bragg and the author [3-6], transforms (1.1) and (1.2) take on new significance, since they are exactly the transforms that relate important problems in partial differential equations. Let $x=\left(x_{1}, x_{2}, \ldots, x_{n}\right)$ and $\Delta_{n}$ be the $n$-dimensional Laplacian. $\neq$ Let $v(x, t)$ be a solution of the wave equation $v_{t t}=\Delta_{n} v$ satisfying $v(x, 0)=\phi(x)$ and $v_{t}(x, 0)=0$. Then under suitable hypotheses

$$
u(x, t)=\frac{1}{\sqrt{(\pi t)}} \int_{0}^{\infty} e^{-s^{2} / 4 t} v(x, s) d s
$$

is a solution of the heat equation $u_{t}=\Delta_{n} u$ satisfying $u(x, 0)=\phi(x)$. Furthermore, under appropriate conditions

$$
w(x, t)=\frac{2 t}{\pi} \int_{0}^{\infty} \frac{v(x, s)}{s^{2}+t^{2}} d s
$$

is a solution of Laplace's equation $w_{t t}+\Delta_{n} w=0$ satisfying $w(x, 0)=\phi(x)$. As we shall see, the relations which Widder derives $[1,2]$, now become important formulas in relating solutions of partial differential equations.

In reference [7], we showed that the heat equation and Laplace's equation are related by the transform (1.3). Indeed, if $u(x, t)$ is a solution of the initial value problem for the heat

$\dagger$ This research was supported by N.S.F. Grant GP-8123.

$\ddagger$ More general linear operators than $\Delta_{n}$ are possible. See references [3-7] for details. Also boundary value problems can be considered.

c 
equation mentioned above, then under suitable restrictions

$$
w(x, t)=\frac{t}{\sqrt{ }(4 \pi)} \int_{0}^{\infty} e^{-t^{2} / 4 s} s^{-3 / 2} u(x, s) d s
$$

is a solution of the corresponding Dirichlet problem for Laplace's equation.

In this paper, we show that transform (1.3) is a convolution transform closely related to transform (1.1), and therefore yields to analysis similar to that of Widder. We obtain inversion theorems for transform (1.3) and show how these and similar theorems for (1.1) and (1.2) apply to related problems in partial differential equations.

2. Complex inversion theory. In this section, we briefly outline the complex inversion theory for the transforms (1.1), (1.2), and (1.3). In transforming classical solutions of partial differential equations we shall generally have functions which are continuous as functions of $t$ and have continuous first partial derivatives with respect to $t$. Therefore, we do not state our results in quite the generality possible (see [8]).

In (1.1) assume that $g(s)$ is continuous and has a continuous first derivative for $s \geqq 0$ and is $O\left(e^{a s^{2}}\right)$ as $s \rightarrow \infty$. Then, making the change of variables $\sigma=s^{2}$ in (1.1), we have

$$
\sqrt{ }(4 \pi t) f(t)=\int_{0}^{\infty} e^{-\sigma / 4 t}\{g(\sqrt{ } \sigma) / \sqrt{ } \sigma\} d \sigma
$$

and $g(\sqrt{ } \sigma) / \sqrt{ } \sigma=O\left(e^{a \sigma}\right)$ as $\sigma \rightarrow \infty$. Therefore

$$
F(z)=\sqrt{ }(4 \pi z) f(z)=\int_{0}^{\infty} e^{-\sigma / 4 z}\{g(\sqrt{ } \sigma) / \sqrt{ } \sigma\} d \sigma
$$

is analytic for $\operatorname{Re}(1 / 4 z)>a$, and the complex inversion formula applies. Hence, letting $\zeta=1 / 4 z$, we have, for $\gamma>a$,

or, assuming $t>0$,

$$
\frac{g(\sqrt{ } \sigma)}{\sqrt{ } \sigma}=\frac{1}{2 \pi i} \int_{\gamma-i \infty}^{\gamma+i \infty} F\left(\frac{1}{4 \zeta}\right) e^{\sigma \zeta} d \zeta
$$

$$
g(t)=\frac{t}{2 i \sqrt{ } \pi} \int_{\gamma-i \infty}^{\gamma+i \infty} \zeta^{-\frac{1}{2}} f\left(\frac{1}{4 \zeta}\right) e^{t^{2} \zeta} d \zeta .
$$

In (1.2) let us assume that $g(s)$ is continuous for $0 \leqq s<\infty$ and $g(s) /\left(1+s^{2}\right)$ belongs to $L(0, \infty)$. Then

$$
f(z)=\frac{2 z}{\pi} \int_{0}^{\infty} \frac{g(s)}{s^{2}+z^{2}} d s
$$

is analytic for $\operatorname{Re} z>0$. Let $\varepsilon>0, t>0$ and pick branches of $\sqrt{ }\left(-t^{2}-i \varepsilon\right)$ and $\sqrt{ }\left(-t^{2}+i \varepsilon\right)$ with values in the right half-plane $\operatorname{Re} z>0$. Then

$$
\frac{t}{2 i}\left[\frac{f\left(\sqrt{ }\left(-t^{2}-i \varepsilon\right)\right)}{\sqrt{ }\left(-t^{2}-i \varepsilon\right)}-\frac{f\left(\sqrt{ }\left(-t^{2}+i \varepsilon\right)\right)}{\sqrt{ }\left(-t^{2}+i \varepsilon\right)}\right]=\frac{2 t \varepsilon}{\pi} \int_{0}^{\infty} \frac{g(s)}{\left(s^{2}-t^{2}\right)^{2}+\varepsilon^{2}} d s
$$


In the integral we let $s^{2}=\eta$, and then

$$
\frac{2 t \varepsilon}{\pi} \int_{0}^{\infty} \frac{g(s)}{\left(s^{2}-t^{2}\right)^{2}+\varepsilon^{2}} d s=\frac{t \varepsilon}{\pi} \int_{0}^{\infty} \frac{g(\sqrt{ } \eta) / \sqrt{ } \eta}{\left(\eta-t^{2}\right)^{2}+\varepsilon^{2}} d \eta .
$$

Since $g(\sqrt{ } \eta) /\left\{\left(1+\eta^{2}\right) \sqrt{ } \eta\right\}$ is integrable and continuous for $\eta>0$, we can take the limit as $\varepsilon \rightarrow 0+$ and we obtain the inversion formula for $t>0$

$$
g(t)=\lim _{t \rightarrow 0+} \frac{t}{2 i}\left[\frac{f\left(\sqrt{ }\left(-t^{2}-i \varepsilon\right)\right)}{\sqrt{\left(-t^{2}-i \varepsilon\right)}}-\frac{f\left(\sqrt{ }\left(-t^{2}+i \varepsilon\right)\right)}{\sqrt{ }\left(-t^{2}+i \varepsilon\right)}\right] .
$$

In (1.3) assume that $g(s)$ is continuous and has a continuous derivative for $t \geqq 0$ and is $O\left(s^{\alpha}\right), \alpha<\frac{1}{2}$, as $s \rightarrow \infty$. Letting $s=1 / 4 \eta$, we have

$$
\frac{\sqrt{ }(\pi) f(t)}{t}=\int_{0}^{\infty} e^{-t^{2} \eta} \eta^{-\frac{t}{2}} g(1 / 4 \eta) d \eta,
$$

where $g(1 / 4 \eta)=O\left(\eta^{-\alpha}\right),-\alpha>-\frac{1}{2}$ as $\eta \rightarrow 0$. Therefore

$$
F(z)=\frac{\sqrt{ }(\pi) f(z)}{z}=\int_{0}^{\infty} e^{-z^{2} \eta} \eta^{-\frac{1}{2}} g(1 / 4 \eta) d \eta
$$

is analytic for $\operatorname{Re} z^{2}>0$. The complex inversion formula is valid. Hence, for $\xi=z^{2}, y>0$,

$$
\eta^{-\frac{1}{2}} g(1 / 4 \eta)=\frac{\sqrt{ } \pi}{2 \pi i} \int_{\gamma-i \infty}^{\gamma+i \infty} \xi^{-\frac{1}{2}} f\left(\xi^{\frac{1}{t}}\right) e^{\eta \xi} d \xi
$$

If $t>0$ and $\eta=1 / 4 t$,

$$
g(t)=\frac{1}{4 i \sqrt{ }(\pi t)} \int_{\gamma-i \infty}^{\gamma+1 \infty} \xi^{-\frac{1}{2}} f\left(\xi^{\frac{1}{3}}\right) e^{\xi / 4 t} d \xi
$$

3. Real inversion theory. All three transforms (1.1), (1.2) and (1.3) can be expressed as convolution transforms with kernels which are of the type treated by Hirschman and Widder [9]. This leads to the following real inversion formulas.

In (1.1) let $t=e^{-x}, s=2 e^{-y / 2}$. Then

$$
f\left(e^{-x}\right)=\frac{1}{\sqrt{ } \pi} \int_{-\infty}^{\infty} e^{-e^{x-y}} e^{(x-y) / 2} g\left(2 e^{-y / 2}\right) d y=\int_{-\infty}^{\infty} G(x-y) \phi(y) d y,
$$

where $G(y)=(1 / \sqrt{ } \pi) e^{-e y} e^{y / 2}$ and $\phi(y)=g\left(2 e^{-y / 2}\right)$. To find the inversion operator we compute (following Hirschman and Widder) the bilateral Laplace transform of $G(y)$.

Then

$$
\frac{1}{E(s)}=\int_{-\infty}^{\infty} e^{-s y} G(y) d y=\frac{1}{\sqrt{\pi}} \Gamma\left(\frac{1}{2}-s\right) \text {. }
$$

$$
E(s)=\frac{\sqrt{ } \pi}{\Gamma\left(\frac{1}{2}-s\right)}=\lim _{n \rightarrow \infty} n^{s} \prod_{k=1}^{n}\left(1-\frac{2 s}{2 k-1}\right) .
$$


Let $D=d / d x$ and define the symbolic operator

$$
E(D)=\lim _{n \rightarrow \infty} n^{D} \prod_{k=1}^{n}\left(1-\frac{2 D}{2 k-1}\right) .
$$

Then, under appropriate conditions, the following inversion is possible

$$
g\left(2 e^{-x / 2}\right)=E(D) f\left(e^{-x}\right) .
$$

Taking into account the changes of variables, we define $\theta F(x)=-x F^{\prime}(x)$, and

$$
L_{n, x}[f]=n^{\theta} \prod_{k=1}^{n}\left(1-\frac{2 \theta}{2 k-1}\right) f(x)
$$

Then, if $g(s)$ is continuous and (1.1) converges for $0<t<r$, we have, for $x>0$,

$$
g(\sqrt{ } 4 x)=\lim _{n \rightarrow \infty} L_{n, x}[f] \text {. }
$$

In (1.2) let $g(s)=s h(s)$ and make the changes of variables $t=e^{-x}, s=e^{-\eta}$. Then

$$
F\left(e^{-x}\right)=e^{x} f\left(e^{-x}\right)=\frac{2}{\pi} \int_{-\infty}^{\infty} \frac{h\left(e^{-\eta}\right)}{e^{-2(x-\eta)}+1} d \eta=\int_{-\infty}^{\infty} G(x-\eta) \phi(\eta) d \eta,
$$

where

$$
G(\eta)=\frac{2}{\pi} \frac{1}{e^{-2 \eta}+1}, \quad \phi(\eta)=h\left(e^{-\eta}\right) .
$$

Taking the two-sided Laplace transform of $G(\eta)$, we have

$$
\frac{1}{E(s)}=\frac{2}{\pi} \int_{-\infty}^{\infty} \frac{e^{-s \eta}}{e^{-2 \eta}+1} d \eta=\frac{1}{\sin \pi s / 2} .
$$

If $D=d / d x$, then the inversion operator is

$$
E(D)=\sin \frac{\pi D}{2}=\frac{\pi}{2} \lim _{n \rightarrow \infty} D \prod_{k=1}^{n}\left(1-\frac{D^{2}}{4 k^{2}}\right) \text {. }
$$

To take into account the changes of variable, we define $\tilde{\theta} G(x)=x G^{\prime}(x)$, and the operator

$$
\tilde{L}_{n, x}[f]=-\tilde{\theta} \prod_{k=1}^{n}\left(1-\frac{\tilde{\theta}^{2}}{4 k^{2}}\right) f(x)
$$

Then, if $g(s) / s$ is continuous for $0<s<\infty$ and belongs to $L(0, \infty)$, and the integral (1.2) converges for some $t>0$,

$$
g(x)=\frac{\pi x}{2} \lim _{n \rightarrow \infty} \tilde{L}_{n, x}[f(x) / x],
$$

the formula holding for all positive $x$. 
In (1.3) let $t=e^{x / 2}$ and $s=e^{y / 4}$. Then

$$
f\left(e^{x / 2}\right)=\frac{1}{\sqrt{ } \pi} \int_{-\infty}^{\infty} e^{-e^{x-y}} e^{(x-y) / 2} g\left(\frac{e^{y}}{4}\right) d y=\int_{-\infty}^{\infty} G(x-y) \phi(y) d y,
$$

where $G(y)=(1 / \sqrt{ } \pi) e^{-e^{y}} e^{y / 2}$ and $\phi(y)=g\left(e^{y} / 4\right)$. We see that we have the same kind of convolution transform as in (1.1). Hence

$$
\frac{1}{E(s)}=\int_{-\infty}^{\infty} e^{-s y} G(y) d y=\frac{1}{\sqrt{ } \pi} \Gamma\left(\frac{1}{2}-s\right) .
$$

To take into account the changes of variable, we define $\bar{\theta} F(x)=\frac{1}{2} x F^{\prime}(x)$, and the inversion operator

$$
\bar{L}_{n, x}[f]=n^{\bar{\theta}} \prod_{k=1}^{n}\left(1-\frac{2 \bar{\theta}}{2 k-1}\right) f(x)
$$

We shall prove the following formula for the inversion of (1.3):

$$
g\left(\frac{x^{2}}{4}\right)=\lim _{n \rightarrow \infty} \bar{L}_{n, x}[f] \text {. }
$$

We first note that $\theta\left(x^{p}\right)=\frac{1}{2} x p x^{p-1}=\frac{1}{2} p x^{p}, \bar{\theta}^{2}\left(x^{p}\right)=\left(\frac{1}{2} p\right)^{2} x^{p}$, etc. We therefore interpret

$$
n^{\bar{\theta}}\left(x^{p}\right)=n^{p / 2} x^{p}=(x \sqrt{ } n)^{p}
$$

and

$$
n^{\bar{\theta}} F(x)=F(x \sqrt{ } n) .
$$

It is a straightforward calculation to show that, if

$$
k(t, s)=\frac{t}{\sqrt{(2 \pi)}} e^{-t^{2} / 4 s}
$$

then, operating on $k$ with respect to $t$, we obtain

$$
\prod_{k=1}^{n}\left(1-\frac{2 \bar{\theta}}{2 k-1}\right) k(t, s)=\frac{n ! t^{2 n+1} e^{-t^{2} / 4 s}}{\sqrt{ }(4 \pi)(2 n) ! s^{n}} .
$$

Therefore, using (3.4), we have

$$
L_{n, t}[k(t, s)]=\frac{n ! n^{\frac{1}{2}(2 n+1)} t^{2 n+1} e^{-n t^{2} / 4 s}}{\sqrt{ }(4 \pi)(2 n) ! s^{n}} .
$$

We have seen above that if $g(s)$ in (1.3) is continuous for $0 \leqq t<\infty$ and $O\left(s^{\alpha}\right)$ for $\alpha<\frac{1}{2}$, as $s \rightarrow \infty$, then $\sqrt{ }(\pi) f(t) / t$ is a Laplace transform. Therefore the integral converges for $0<t<\infty$ and the differentiation under the integral sign is justified. Hence

$$
L_{n, t}[f(t)]=\frac{n ! n^{\frac{1}{2}(2 n+1)} t^{2 n+1}}{\sqrt{ }(4 \pi)(2 n) !} \int_{0}^{\infty} e^{-n t^{2} / 4 s} s^{-\left(n+\frac{2}{2}\right)} g(s) d s .
$$


Letting $u=1 / s$, we have

$$
L_{n, 1}[f(t)]=\frac{n ! n^{\frac{1}{2(2 n+1)} t^{2 n+1}}}{\sqrt{(4 \pi)(2 n) !}} \int_{0}^{\infty} e^{-n u t^{2} / 4} u^{n-\frac{1}{2}} g\left(\frac{1}{u}\right) d u,
$$

and an application of the Laplace asymptotic method [8] shows that

$$
\lim _{n \rightarrow \infty} \bar{L}_{n, t}[f(i)]=g\left(\frac{1}{4} t^{2}\right)
$$

which proves formula (3.3).

4. Expansion theorems. The inversion theorems using series proved by Widder in [1] and [2] now become expansion theorems for solutions of partial differential equations. For example, we have

THEOREM 4.1. Let $v(x, t)$ be a solution of the wave equation $v_{t}=\Delta_{n} v$ in some domain $D \subset \mathbf{R}^{n}$, satisfying $v(x, 0)=\phi(x)$ and $v_{t}(x, 0)=0$, such that $v(x, t)=O\left(e^{a t^{2}}\right)$ as $t \rightarrow \infty$ for $x \in D$. Then

$$
u(x, t)=\frac{1}{\sqrt{ }(\pi t)} \int_{0}^{\infty} e^{-s^{2} / 4 t} v(x, s) d s
$$

is a solution of the heat equation $u_{t}=\Delta_{n} u$ in $D$ satisfying $u(x, 0)=\phi(x)$. If

$$
u(x, t)=\sum_{k=0}^{\infty} a_{k}(x) t^{k}
$$

for $x \in D, 0 \leqq t<\sigma$, then

$$
v(x, t)=\sqrt{ } \pi \sum_{k=0}^{\infty} \frac{a_{k}(x) t^{2 k}}{4^{k} \Gamma\left(k+\frac{1}{2}\right)}
$$

for $0 \leqq t<\infty$. If, for some real numbers $\alpha$ and $c, u(x, t)$ has an expansion of the form

$$
u(x, t)=\sum_{k=0}^{\infty} b_{k}(x) t^{-k-\alpha}
$$

for $0<t<\infty$, where $b_{k}(x)=O\left(c^{k} / k !\right)$ as $k \rightarrow \infty$ for all $x \in D$, then

$$
v(x, t)=\sqrt{ } \pi \sum_{k=0}^{\infty} \frac{b_{k}(x) 4^{k+\alpha}}{\Gamma\left(\frac{1}{2}-k-\alpha\right) t^{2 k+2 \alpha}}
$$

for $4 c e^{\pi}<t^{2}<\infty$

Similarly, in connection with transform (1.2) we have the following theorem:

THEOREM 4.2. Let $v(x, t)$ be a solution of the wave equation $v_{t}=\Delta_{n} v$ in some domain $D \subset \mathbf{R}^{n}$, satisfying $v(x, 0)=\phi(x)$ and $v_{t}(x, 0)=0$, such that $v(x, t) /\left(1+t^{2}\right) \in L(0, \infty)$ with respect to $t$ for each $x \in D$. Then 


$$
w(x, t)=\frac{2 t}{\pi} \int_{0}^{\infty} \frac{v(x, s)}{s^{2}+t^{2}} d s
$$

is a solution of Laplace's equation $w_{n}+\Delta_{n} w=0$ in D satisfying $w(x, 0)=\phi(x)$. If

$$
w(x, t)=\sum_{k=0}^{\infty} a_{k}(x) t^{k}
$$

for $0 \leqq t<\rho, x \in D$, then

$$
v(x, t)=\sum_{k=0}^{\infty}(-1)^{k} a_{2 k} t^{2 k}
$$

for some interval $0 \leqq t<\sigma$. Furthermore, if for some real $\alpha$

$$
w(x, t)=\sum_{k=0}^{\infty} b_{k}(x) t^{-k-\alpha}
$$

for $\rho<t<\infty, x \in D$, then

$$
v(x, t)=\cos \frac{\pi \alpha}{2} \sum_{k=0}^{\infty} \frac{(-1)^{k} b_{2 k}(x)}{t^{2 k+\alpha}}-\sin \frac{\pi \alpha}{2} \sum_{k=0}^{\infty} \frac{(-1)^{k} b_{2 k+1}(x)}{t^{2 k+\alpha+1}}
$$

in some interval $\sigma<t<\infty$.

Finally, we prove an expansion theorem in reference to transform (1.3).

THEOREM 4.3. Let $u(x, t)$ be a solution of the heat equation $u_{t}=\Delta_{n} u$ in some domain $D \subset \mathbf{R}^{n}$, satisfying $u(x, 0)=\phi(x)$ and such that $u(x, t)=O\left(t^{\alpha}\right)$ as $t \rightarrow \infty\left(\alpha<\frac{1}{2}\right)$ for all $x \in D$. Then

$$
w(x, t)=\frac{t}{\sqrt{ }(4 \pi)} \int_{0}^{\infty} e^{-t^{2} / 4 s} s^{-\frac{1}{3}} u(x, s) d s
$$

is a solution of Laplace's equation $w_{t t}+\Delta_{n} w$ in D satisfying $w(x, 0)=\phi(x)$. If

$$
w(x, t)=\sum_{k=0}^{\infty} a_{k}(x) t^{k}
$$

for $x \in D$ and $0 \leqq t<\infty$, where $a_{k}(x)=O\left(c^{k} / k !\right)$ as $k \rightarrow \infty$ for some positive $c$, then

$$
u(x, t)=\sqrt{ } \pi \sum_{k=0}^{\infty} \frac{4^{k} a_{2 k}(x) r^{k}}{\Gamma\left(\frac{1}{2}-k\right)}
$$

for $0 \leqq t<\infty$. If, for some $\alpha$,

$$
w(x, t)=\sum_{k=0}^{\infty} b_{k}(x) t^{-k-\alpha}
$$

for $x \in D$ and $\sigma<t<\infty$, then

$$
u(x, t)=\sqrt{ } \pi \sum_{k=0}^{\infty} \frac{b_{k}(x) t^{-(k+\alpha) / 2}}{2^{\alpha+k} \Gamma\left(\frac{1}{2}(k+\alpha+1)\right)}
$$

for $0<t<\infty$. 
Proof. The proof that $w(x, t)$ is a solution of the Dirichlet problem is contained in reference [6].

It is easily verified that

$$
n^{\bar{\theta}} \prod_{j=1}^{n}\left(1-\frac{2 \bar{\theta}}{2 j-1}\right) t^{k}=\left\{\begin{array}{cc}
S_{n}\left(\frac{1}{2} k\right), & k \text { even }, \\
0, & k \text { odd }
\end{array}\right.
$$

where

$$
S_{n}(z)=n^{2} \prod_{j=1}^{n}\left(1-\frac{2 z}{2 j-1}\right) .
$$

Therefore, since we are permitted to differentiate a power series term by term,

$$
\bar{L}_{n, t}\left[\sum_{k=0}^{\infty} a_{k}(x) t^{k}\right]=\sum_{k=0}^{\infty} S_{n}(k) a_{2 k}(x) t^{2 k} .
$$

By Lemma 5.4 of $[1],\left|S_{n}(k)\right| \leqq \pi^{-\frac{1}{2}} \Gamma\left(\frac{1}{2}+k\right) \cosh \pi k$. We take as a comparison series

$$
\frac{1}{\sqrt{ } \pi} \sum_{k=0}^{\infty}\left|a_{2 k}(x)\right| t^{2 k} \Gamma\left(\frac{1}{2}+k\right) e^{\pi k}
$$

or, since $\Gamma\left(\frac{1}{2}+k\right)=[\sqrt{ } \pi \cdot(2 k) !] / 2^{2 k} k !$, the series

$$
\sum_{k=0}^{\infty} \frac{c^{2 k} t^{2 k} e^{\pi k}}{2^{2 k} k !}
$$

By the ratio test, this series converges for $0 \leqq t<\infty$. Therefore, since the convergence is uniform in $n$,

$$
u\left(x, t^{2} / 4\right)=\lim _{n \rightarrow \infty} L_{n, t}[w(x, t)]=\sqrt{ } \pi \sum_{k=0}^{\infty} \frac{a_{2 k}(x) t^{2 k}}{\Gamma\left(\frac{1}{2}-k\right)}
$$

and, replacing $t^{2} / 4$ by $t$, we have

$$
u(x, t)=\sqrt{ } \pi \sum_{k=0}^{\infty} \frac{4^{k} a_{2 k}(x) t^{k}}{\Gamma\left(\frac{1}{2}-k\right)}
$$

for $0 \leqq t<\infty$.

For the last part of the theorem, we note that

$$
n^{\bar{\theta}} \prod_{j=1}^{n}\left(1-\frac{2 \bar{\theta}}{2 j-1}\right) t^{-k-\alpha}=S_{n}\left(-\frac{k}{2}-\frac{\alpha}{2}\right) t^{-k-\alpha}
$$

and, taking derivatives term by term, we have

$$
L_{n, t}\left[\sum_{k=0}^{\infty} b_{k}(x) t^{-k-\alpha}\right]=\sum_{k=0}^{\infty} b_{k}(x) S_{n}\left(-\frac{k}{2}-\frac{\alpha}{2}\right) t^{-k-\alpha} .
$$


However, now, because of the factor $n^{-(k+\alpha) / 2}$ in $S_{n}\left(-\frac{1}{2} k-\frac{1}{2} \alpha\right)$, the series converges for $\sigma / \sqrt{ } n<t<\infty$. By Lemma 5.2 of [1], for all $n \geqq m$ and $k \geqq-\alpha$,

$$
0 \leqq S_{n}\left(-\frac{k}{2}-\frac{\alpha}{2}\right) \leqq S_{m}\left(-\frac{k}{2}-\frac{\alpha}{2}\right)
$$

We take as a comparison series

$$
\sum_{k \geqq-\alpha}\left|b_{k}(x)\right| S_{m}\left(-\frac{k}{2}-\frac{\alpha}{2}\right) t^{-k-\alpha},
$$

which converges for $\sigma / \sqrt{ } m<t<\infty$. Therefore, since the convergence is uniform in $n$,

$$
u\left(x, t^{2} / 4\right)=\lim _{n \rightarrow \infty} L_{n, t}[w(x, t)]=\sqrt{ } \pi \sum_{k=0}^{\infty} \frac{b_{k}(x) t^{-k-\alpha}}{\Gamma\left(\frac{1}{2}+\frac{1}{2} k+\frac{1}{2} \alpha\right)} .
$$

However, since $m$ is arbitrary, the resulting series converges for $0<t<\infty$. Finally, replacing $t^{2} / 4$ by $t$, we have

$$
u(x, t)=\sqrt{ } \pi \sum_{k=0}^{\infty} \frac{b_{k}(x) t^{-(k+\alpha) / 2}}{2^{k+\alpha} \Gamma[(k+\alpha+1) / 2]}
$$

\section{REFERENCES}

1. D. V. Widder, Inversion of a heat transform by use of series, J. d'Analyse Math. 18 (1967), $389-413$.

2. D. V. Widder, A transform related to the Poisson integral for a half-plane, Duke Math. J. 33 (1966), 355-362.

3. L. R. Bragg and J. W. Dettman, Related problems in partial differential equations, Bull. Amer. Math. Soc. 74 (1968), 375-378.

4. L. R. Bragg and J. W. Dettman, Related partial differential equations and their applications, S.I.A.M. J. Appl. Math. 16 (1968), 459-467.

5. L. R. Bragg and J. W. Dettman, An operator calculus for related partial differential equations, J. Math. Anal. \& Appl. 22 (1968), 261-271.

6. J. W. Dettman, Initial boundary-value problems related through the Stieltjes transform, $J$. Math. Anal. \& Appl. 25 (1969), 341-349.

7. L. R. Bragg and J. W. Dettman, A class of related Dirichlet and initial value problems, Proc. Amer. Math. Soc. 21 (1969), 50-56.

8. D. V. Widder, The Laplace transform (Princeton, 1946).

9. I. I. Hirschman and D. V. Widder, The convolution transform (Princeton, 1955).

OAKLAND UNIVERSITY

ROCHESTER, MichIGAN 